\title{
Comparison of the plasma temperature and electron number density of pulsed electrolyte cathode atmospheric pressure discharge and direct current solution cathode glow discharge
}

Jinmei Wang, Shiyu Li, Peichao Zheng * and Yanying Chen

Chongqing Municipal Level Key Laboratory of Photoelectronic Information Sensing and Transmitting Technology, College of Optoelectronic Engineering, Chongqing University of Posts and Telecommunications; Chongqing 400065, China

*Correspondence: zhengpc@cqupt.edu.cn ;Tel: +86-23-62460592.

Abstract: A novel plasma source of pulsed electrolyte cathode atmospheric pressure discharge (pulsed-ECAD) driven by an alternating current $(\mathrm{AC})$ power supply coupled with a high voltage diode was generated, and the discharge was generated in the open-to-air atmosphere between a metal electrode and a small-sized flowing liquid cathode. The spatial distribution of plasma temperatures (excitation, vibrational and rotational) of the pulsed-ECAD were investigated. The electron excitation temperature of $\mathrm{H} T_{\text {exc }}(\mathrm{H})$, vibrational temperature of $\mathrm{N}_{2} T_{\text {vib }}\left(\mathrm{N}_{2}\right)$, and rotational temperature of $\mathrm{OH}$ $T_{\text {rot }}(\mathrm{OH})$ were measured as $4900 \pm 36-6800 \pm 108 \mathrm{~K}, 4600 \pm 86-5800 \pm 100 \mathrm{~K}$ and $1050 \pm 20-1140 \pm 10 \mathrm{~K}$, respectively. Meanwhile, the temperature characteristics of dc solution cathode glow discharge (dc-SCGD) were also studied for comparison with pulsed-ECAD. The effects of operating parameters, including discharge voltage and discharge frequency, on the plasma temperatures were investigated. The electron number density determined in the discharge system and dc-SCGD were within the range $(3.8-18.9) \times 10^{14} \mathrm{~cm}^{-3}$ and $2.6 \times 10^{14}-17.2 \times 10^{14} \mathrm{~cm}^{-3}$, respectively.

Keywords: pulsed electrolyte cathode atmospheric pressure discharge (pulsed-ECAD); plasma temperatures; temperature characteristics; electron number density

\section{Introduction}

Glow discharge generated with solution-electrodes, a type of atmospheric pressure glow discharge where one or both electrodes is a flowing liquid, has enjoyed increasing interest over recent years, because of its potential application in analytical atomic spectroscopy [1,2]. The first solution electrode system used for analytical atomic spectroscopy was developed in 1993 by Cserfalvi et al., who later named source an electrolyte cathode atmospheric pressure glow discharge (ELCAD)[3]. Since then multiple sources based on a liquid electrode have been investigated, including the liquid sampling-atmospheric pressure glow discharge (LS-APGD) [4,5], liquid electrode plasma-Atomic emission spectrometry (LEP-AES) [6], liquid-film dielectric barrier discharge (LFDBD)[7] and liquid electrode spectral emission chip[8-11]. In 2005, a simple configuration of ELCAD was described by Webb et al. and given the name solution cathode glow discharge (SCGD), and this showed better analytical performance than the previous system [12]. The SCGD or other ELCAD-like sources offered important advantages over conventional plasma sources for solution analysis, such as inductively 
coupled plasma-mass spectrometry (ICP-MS) [13] and inductively coupled plasma-atomic emission spectrometry (ICP-AES) [14]. Several reviews [15-20] detailed the development of all these discharges. The SCGD source was both inexpensive and simple, requiring no compressed gases or sample-solution nebulizer. It operated at lower power $(\sim 70 \mathrm{~W})$, and exhibited lower spectral interference [21]. In order to evaluate the analytical performance of these excitation sources, their plasma parameters, including the electron excitation temperature, vibrational temperature, rotational temperature and electron number density, were investigated. The spatial distribution of the rotational temperature of $\mathrm{OH}$ (4000-8000 K) and the excitation temperature of $\mathrm{Cu}(5500-7500 \mathrm{~K})$ were reported by Cservalvi et al. in an electrolyte cathode-atmospheric pressure discharge (ELCAD) source [22]. The excitation temperature of $\mathrm{Fe}$ and rotational temperature of $\mathrm{OH}$ as well as the emission intensities of the main species were investigated by Webb et al. [23,24] as a function of distance from the liquid cathode. Maximum temperatures and emission intensities were observed near the cathode region. Bruggeman et al. $[25,26]$ reported the rotational temperatures of $\mathrm{OH}$ and $\mathrm{N}_{2}$, the electron number density and the excitation temperature of $\mathrm{H}$ achieved using distilled water and a solution of $\mathrm{NaOH}$ and $\mathrm{NaCl}$ as liquid cathodes. Jamróz et al.[27,28] studied the effect of gas flow rate on spectroscopic temperatures (rotational, vibrational and excitation) in a miniaturized atmospheric pressure glow microdischarge system ( $\mu$ APGD) under conditions of a changing flow rate of $\operatorname{Ar}(\sim 300 \mathrm{sccm})$ and $\mathrm{He}(30-300 \mathrm{sccm})$, respectively. The density and temperature of electrons and metal elements in an atmospheric-pressure pulsed-dc helium discharge plasma were measured by Naoki et al. [29] and the results showed that the electron density and temperature varied from $2.4 \times 10^{14} \mathrm{~cm}^{-3}$ and $1.8 \mathrm{eV}$ in the center of the discharge to $0.8 \times 10^{14} \mathrm{~cm}^{-3}$ and $1.5 \mathrm{eV}$ near the outer edge of the plasma in the case of a metal anode. Li et al. [30] also measured the rotational temperature of $\mathrm{OH}$ in miniature flow $\mathrm{Ar}$, $\mathrm{He}$ or $\mathrm{N}_{2}$ dc discharges with a bulky liquid electrode. The rotational temperature of $\mathrm{OH}$ established for the He microjet was higher than the Ar microjet system.

Recently, a pulsed Electrolyte Cathode Atmospheric Pressure Discharge (pulsed-ECAD) was developed in our lab [31]. Unlike the dc-SCGD, the pulsed-ECAD was driven by an alternating current (AC) power supply coupled with a high voltage diode. The intensity of the emission spectral band of $\mathrm{OH}$ obtained in the pulsed-ELCD was far less than those in SCGD. The background signal intensities and SDs of the background signals were much lower than dc-SCGD's. Clearly, there were still some differences in the chemistry and physics of the processes occurring in these sources. The knowledge gained from fundamental studies is helpful to explain the emission mechanisms and led to the potential to further improve analytical perfomance, as earlier studies had.

The aim of this work was the spectroscopic characterization of the pulsed electrolyte cathode plasma driven by an alternating-current power supply coupled with a high voltage diode. Various plasma parameters were measured in various experimental conditions. The parameters investigated included the electron excitation temperature $T_{\text {exc }}(\mathrm{H})$, the vibrational temperature $T_{\text {vib }}\left(\mathrm{N}_{2}\right)$ and the rotational temperature $T_{\text {rot }}(\mathrm{OH})$, as well as the electron number density $n_{e}$. These plasma parameters were measured and compared in the pulsed-ECAD and dc-SCGD systems. The possible plasma processes responsible for the excitation of the pulsed-ECAD are discussed here.

\section{ECAD cell design}

The experimental setup has been described previously [31] and is shown in Fig. 1. A glass capillary, which is a $25 \mu \mathrm{L}$ micropipette (SAIHUA) with a $0.35 \mathrm{~mm}$ inner diameter and a $1.38 \mathrm{~mm}$ outer diameter, was used for the sampling inlet. An additional graphite tube with a diameter of $4 \mathrm{~mm}$ 
was inserted axially by the glass capillary, $3 \mathrm{~mm}$ lower than the top of the glass capillary. The overflow from the capillary tip created an electrical connection between the solution exiting the micropipette and the graphite tube. The graphite tube and glass capillary were mounted in a PTFE waste reservoir mounted on a three-dimensional mobile platform in order to adjust the distance between the two electrodes. Sample solutions were introduced into the glass capillary by a peristaltic pump (BT-100, Jieheng, China), and overflowed continuously from the capillary top, falling into the graphite tube. Positioned $1.6 \mathrm{~mm}$ above the capillary tip was a tungsten $\operatorname{rod}\left(\phi 2 \mathrm{~mm} \times 7 \mathrm{~mm}\right.$, pinpoint sloped $\left.45^{\circ}\right)$ that served as the anode for the cell. To generate discharge plasma, an AC power supply (CTP-2000K, Suman Electronics Co., Ltd., Nanjing, China) was applied to the tungsten anode through a high voltage diode in order to cut the negative cycle high voltage. The frequency of the power supply could be changed from $4.7 \mathrm{kHz}$ to $20 \mathrm{kHz}$, the maximum output power being $500 \mathrm{~W}$. The ground of the power supply was connected to the additional graphite tube through a sample resistor of $51 \Omega$ for the discharge current measurement. The applied discharge voltage and discharge frequency were measured using an oscilloscope (Tektronix, DPO 3054) connected to the high-voltage probe (Tektronix, P6015A).

The discharge plasma was imaged 1:1 with a quartz lens onto the optical fiber head. Two spectrometers were used in the experiments. A portable spectrometer (Ocean Optics Maya 2000 Pro) was employed to measure the emission spectrum of discharge and measure the electron excited temperature. The Maya 2000 Pro was a $182 \times 110 \times 47 \mathrm{~mm}$ spectrograph with a CCD detector. The $2048 \times 64$ pixel CCD (Hamamatsu S10420) was backthinned and binned vertically to enhance sensitivity. The configuration used in this study had a spectral range of 200-1100 nm, a 1200 line per $\mathrm{mm}$ grating, a $10 \mu \mathrm{m}$ entrance slit and a spectral resolution of $1.00 \mathrm{~nm}$ FWHM. The experimental parameters for acquiring emission signals were $20 \mathrm{~ms}$ for the integration times and 10 measures in order to increase the stability and reduce the standard deviation of the spectral intensities. A computerized Czerny-Turner spectrometer (Andor SR-750A) was used to capture high resolution spectra for the vibrational temperature, rotational temperature and electron density calculation. The spectrometer was equipped with three ruled gratings: 2400 grooves $/ \mathrm{mm}, 1200$ grooves $/ \mathrm{mm}$ and 300 grooves $/ \mathrm{mm}$, which could be changed under the control of the computer, to provide high and low resolution spectra in the wavelength range of $200-900 \mathrm{~nm}$. A $2048 \times 512$ pixels intensified and gated CCD camera (Andor DH340T-18U-03) was attached to the output end of the spectrograph to detect the spectrally resolved lines. The ICCD camera was cooled to $-15{ }^{\circ} \mathrm{C}$ by a Peltier cooler in order to reduce the noise.

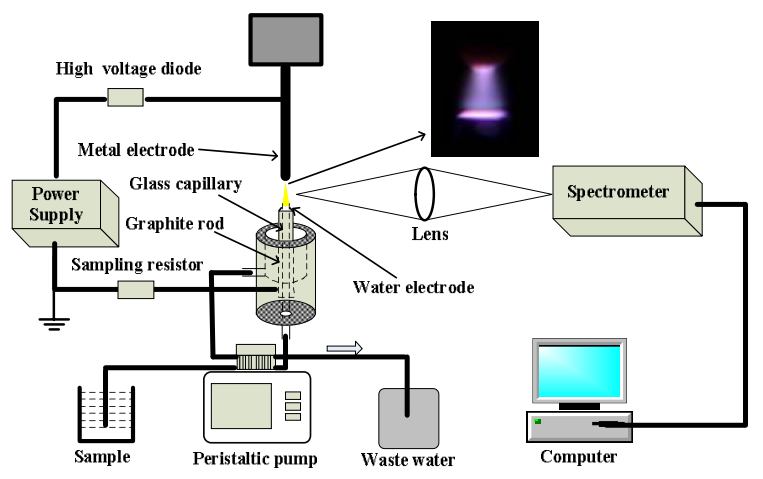

Fig. 1 Schematic diagram of the experimental setup 


\section{Results and discussion}

\subsection{Electrical characteristics}

Fig. 2 presents the waveforms of the discharge voltage and current of the pulsed- ECAD system when the discharge frequency is $4.7 \mathrm{kHz}$. As shown in Fig. 2, the cycle of the discharge is $214 \mu \mathrm{s}$. There is only one pulse in a cycle for both the discharge voltage and current. The duration time of the pulsed discharge voltage is about $0.8 \mu \mathrm{s}$, and the durations time of the pulsed discharge current is about $0.2 \mu \mathrm{s}$. Then the discharge voltage remains roughly unchanged and maintains about $950 \mathrm{~V}$ for about $100 \mu \mathrm{s}$, while the discharge current increases slowly to $180 \mathrm{~mA}$ over about $81.4 \mu \mathrm{s}$. Finally, the discharge voltage decreases to $390 \mathrm{~V}$ at the time $214 \mu$ s. The discharge current reduces to zero at 166 $\mu \mathrm{s}$, and keeps this value until the end of the cycle.

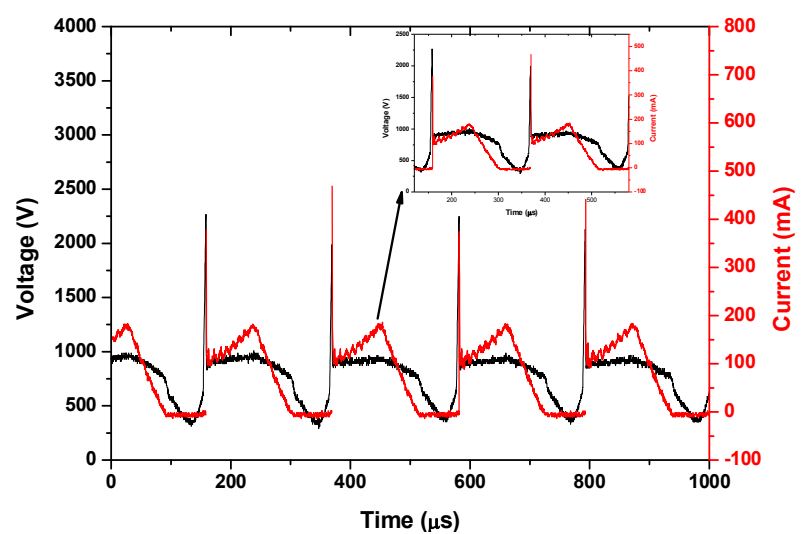

Fig. 2 Voltage and current waveforms of the pulsed-ECAD between metal anode and solution cathode

\subsection{Electron Excitation Temperatures}

The electron excitation temperature is one of the most important temperature parameters in the process of investigating the energy level distribution of the measured atom in the plasma source. It plays an important role in the excitation power of the plasma source. A two-line intensity ratio method was selected for the calculation of excitation temperature. When the thermal equilibrium or the local thermal equilibrium state is satisfied, the number of particles in the two energy states of an atom satisfies the Boltzmann distribution [31]. In our work, $\mathrm{H}_{\alpha} 656.3 \mathrm{~nm}$ and $\mathrm{H}_{\beta} 486.1 \mathrm{~nm}$ lines with excitation energies of 12.1 and $12.7 \mathrm{eV}$, respectively, were chosen for the determination of the excitation temperatures $T_{\text {exc }}(\mathrm{H})$. As shown in Fig. 3, the spectral intensities of $\mathrm{H}_{\alpha} 656.3 \mathrm{~nm}$ and $\mathrm{H}_{\beta}$ $486.1 \mathrm{~nm}$ of pulsed-ECAD system were much lower than dc-SCGD discharge system.
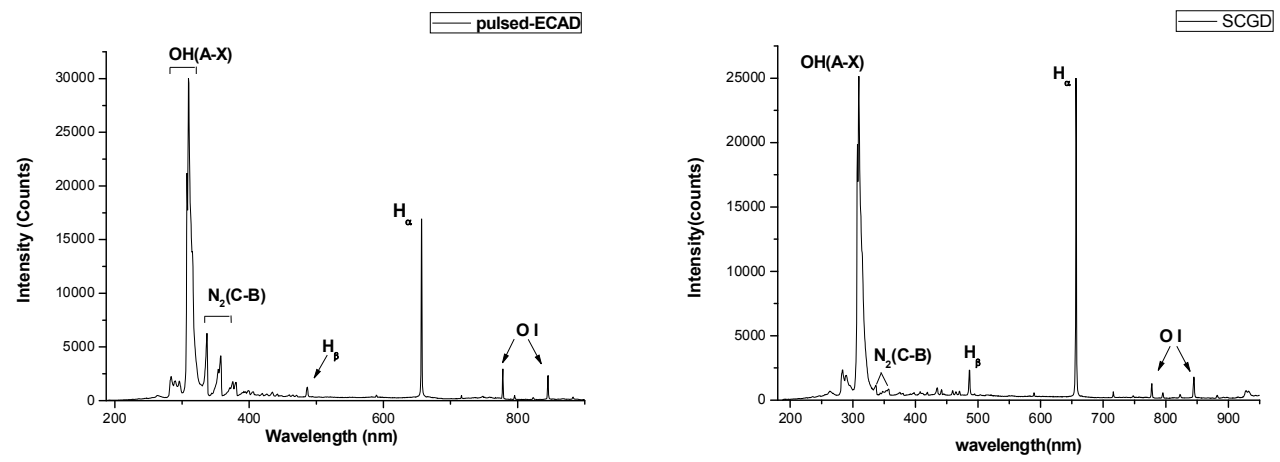

Fig. 3 Emission spectra of (a) pulsed-ECAD and (b) dc-SCGD

The spatial distribution of the electron excitation temperatures was determined using the two-line 
intensity ratio method $\left(\mathrm{H}_{\alpha} 656.3 \mathrm{~nm}\right.$ and $\mathrm{H}_{\beta} 486.1 \mathrm{~nm}$ lines). The electron excitation temperatures were obtained using the discharge systems of pulsed-ECAD and dc-SCGD with the optimal conditions (discharge voltage: $980 \mathrm{~V}$, discharge frequency: $4.7 \mathrm{kHz}$, inter-electrode distance: $1.6 \mathrm{~mm}$, flow rate: $1.75 \mathrm{~mL} \mathrm{~min}^{-1}$ for pulsed-ECAD, inter-electrode distance: $3 \mathrm{~mm}$, flow rate: $2.6 \mathrm{~mL} \mathrm{~min}{ }^{-1}$, discharge voltage: $1 \mathrm{kV}$ for dc-SCGD). The same spectrometer (with the same parameters) and optical capture device were used. The results are presented in Fig. 3. As shown in Fig. 4, the electron excitation temperature of the pulsed-ECAD system (shown in Fig. 4 (a)) was higher near the anode and decreased with the increasing gap to the metal electrode. It is shown that the electron excitation temperature has an upward trend at the near cathode field. However, the electron excitation temperature at the near cathode region of dc-SCGD was weaker than other regions. This may be the reason that the optimal spectral emission positions of some elements ( $\mathrm{Li}, \mathrm{Sr}$ and $\mathrm{Cd}$ ) were not located in the near cathode region [31]. Additionally, the electron excitation temperature near solution electrode was lower than other regions. This phenomenon was different from the electron excitation temperature obtained from the dc-SCGD system (shown in Fig. 4 (b)), which indicated that the electronic excitation ability of pulsed-ECAD at the water level was relatively weaker than that of dc-SCGD. The electron excitation temperatures of the pulsed-ECAD system and dc-SCGD system were in the range 4900 $\pm 36 \sim 6800 \pm 108$ $\mathrm{K}$ and $5700 \pm 5 \sim 19000 \pm 678 \mathrm{~K}$, respectively. Compared to the work of Yoshinobu Kohara[33] whose discharge system (LEP-AES) was driven by a high-voltage DC pulse power, the excitation temperatures of the pulsed-ECAD system were relatively lower than for LEP-AES (8000 K). Additionally, the electron excitation temperature obtained by Peter Bruggeman [25] was 5750-7250 K, slightly higher than the pulsed-ECAD.
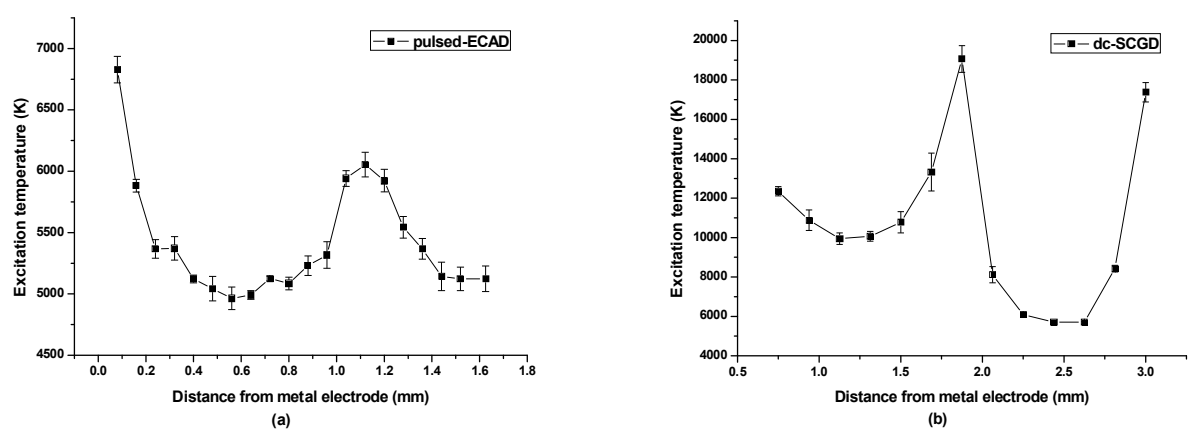

Fig.4 The spatial distributions of the excitation temperatures of the pulsed-ECAD system and dc-SCGD system

\subsection{Vibrational Temperatures}

The vibrational temperature is an important parameter of the plasma. It is related to the activity of the plasma and can reflect the energy exchange information of high energy particles and electrons in the plasma. The vibrational temperature determined from $\mathrm{N}_{2}$ bands is calculated by the Boltzmann plot method, using the four bands of the $\mathrm{C}^{3} \Pi_{\mathrm{u}} \rightarrow \mathrm{B}^{3} \Pi_{\mathrm{g}}$ system, i.e., (0-2); (1-3); (2-4); (3-5) [8].

The Boltzmann slope method using the emission band of $380.4 \mathrm{~nm}(0-2)$ was shown in Fig. 5, and the fitting degree reached to 0.9909 . In our work, the emission band of $N_{2}\left(C^{3} \Pi_{u} \rightarrow B^{3} \Pi_{g}\right)(0-2)$ was selected for the calculation of the vibrational temperature (shown in Fig. 6). 


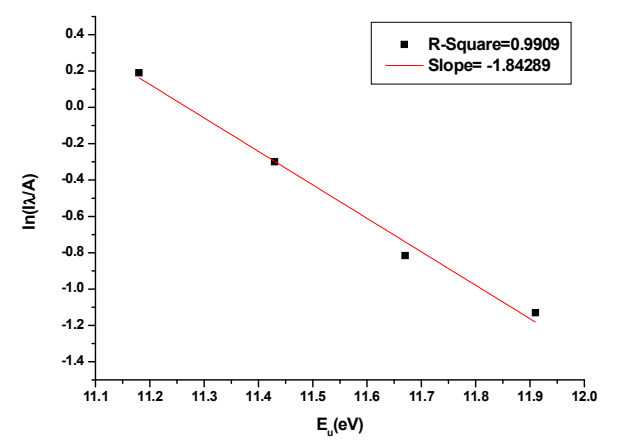

Fig. 5 The Botzmann graph for the rotational states of $\mathrm{N}_{2}(\mathrm{C}-\mathrm{B})$

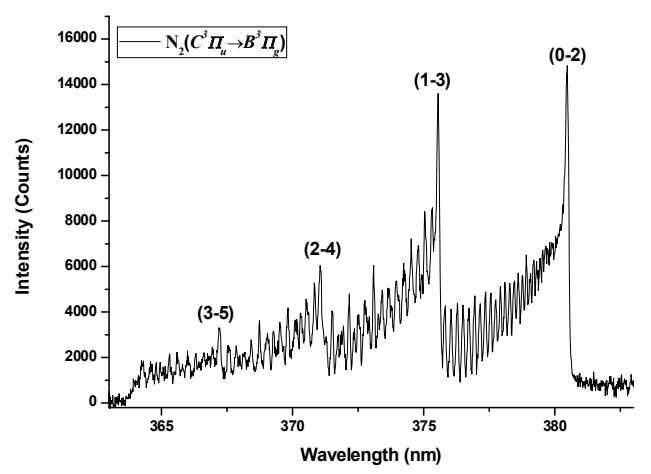

Fig. 6 Emission bands of $\mathrm{N}_{2}\left(C^{3} \Pi_{u} \rightarrow B^{3} \Pi_{g}\right)(0-2)$ in the pulsed-ECAD system

Under the optimal experimental conditions [30], the vibrational temperature of the pulsed-ECAD and dc-SCGD systems were obtained in the range 4600 $\pm 86 \sim 5800 \pm 100 \mathrm{~K}$ and $5150 \pm 200 \sim 13000 \pm 400 \mathrm{~K}$, respectively. The vibrational temperature of pulsed-ECAD was slightly higher than the temperature obtained by Jamróz[8], which was 3800-4200 K. The spatial distribution of the vibrational temperature is presented in Fig. 7. It is obvious that the vibrational temperatures obtained from the two systems had similar spatial distributions. Both vibrational temperatures were high at the middle of the discharge plasmas, which may be because electrons excite gases to ionize and electrons and ions are bipolar diffusion, charged ions are prone to complex reactions. The greater the number of charged particles, the more intense the collisions in this region. As diatomic molecules, the band of $\mathrm{N}_{2}$ is mainly caused by collisions. Therefore, the vibrational temperature of the two discharge systems is higher in the middle region of the plasma. However, the vibrational bands of $\mathrm{N}_{2}$ at the near cathode field of both discharge systems were blurry. These behaviors indicate that the energy exchange between energetic particles and electrons presented in the discharge plasmas is more frequent at the middle of the plasmas than at the near cathode field. 

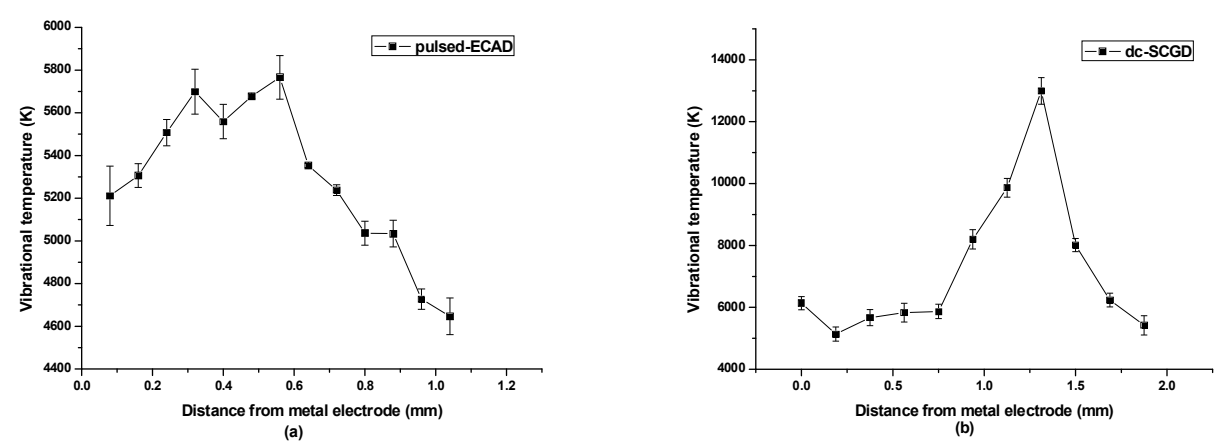

Fig. 7 The spatial distribution of the vibrational temperatures of pulsed-ECAD system and dc-SCGD system

\subsection{Rotational Temperatures}

The rotational temperature is also one of the most important parameters for the plasma. The method to calculate the rotational temperatures was described by Jamróz [8]. For the calculation of the rotational temperature of $\mathrm{OH}\left(\mathrm{T}_{\mathrm{rot}}(\mathrm{OH})\right)$, the (0-0) band of the $\mathrm{OH} \mathrm{A} \mathrm{A}^{2} \rightarrow \mathrm{X}^{2} \Pi$ system with the band head at $309.3 \mathrm{~nm}$ was selected and its emission spectra acquired in the range 307 to $310 \mathrm{~nm}$ (shown in Fig. 8). The Boltzmann slope method using the emission band of $\mathrm{OH}(0-0)$ was shown in Fig. 9, and the fitting degree of the linear fit reached to 0.9844 .

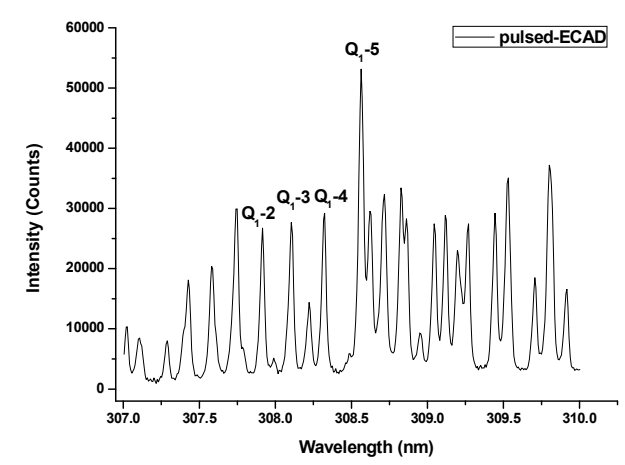

Fig. 8 Emission band of $\mathrm{OH}\left(\mathrm{A}^{2} \Sigma^{+} \rightarrow \mathrm{X}^{2} \Pi\right)(0-0)$ in the pulsed-ECAD system

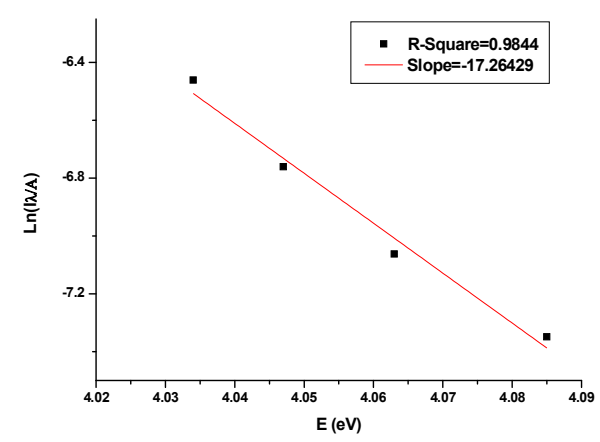

Fig. 9 Fitting Boltzmann diagram slash using the emission band of $\mathrm{OH} A^{2} \Sigma^{+} \rightarrow X^{2} \Pi$ system $(0-0)$

The rotational temperatures of $\mathrm{OH}\left(\mathrm{A}^{2} \Sigma^{+} \rightarrow \mathrm{X}^{2} \Pi\right)(0-0)$ of pulsed-ECAD and dc-SCGD were $1050 \pm 20-1140 \pm 10 \mathrm{~K}$ and $2400 \pm 60-3050 \pm 50 \mathrm{~K}$, respectively. It was shown that the rotational 
temperatures of the pulsed-ECAD and dc-SCGD had the same variation tendency with the increasing of the gap to the metal electrode. The rotational temperature decreased with the increasing of the gap to the metal electrode and had the highest temperature near the anode. A similar rotational temperature was observed by Naoki et al. [29] in pulsed discharge system, and the rotational temperatures of $\mathrm{OH}$ were 1200-1600K. However, the rotational temperature of the discharge system investigated by Peter Bruggeman et al. [26] was in the range 3200-3750 K and the SCGD discharge system investigated by Michael Webb [23] was in the range 2800-3500K. There was three times higher than pulsed-ECAD. Additionally, the rotational temperature of $\mathrm{OH}$ of the discharge system studied by Piotr Jamróz[8] was in the range $3800-4200 \mathrm{~K}$, which was nearly four times higher than the temperature presented here. The behavior of low rotational temperature of pulsed-ECAD indicated that the thermal effect produced by plasma was lower and the evaporation effect of plasma on the solution was relatively weak. The less aerosol generated around the discharge plasmas also indicates the behaivor. Most of the metal ions in the solution may be injected into the plasma due to the cathode sputtering effect. The elements which can be detected by pulsed-ECAD system are alkali metal and alkaline earth metal elements with low demands for excitation energy. For most heavy metals, the excitation energy is too high, and the energy of cathode sputtering is not enough to excite them.
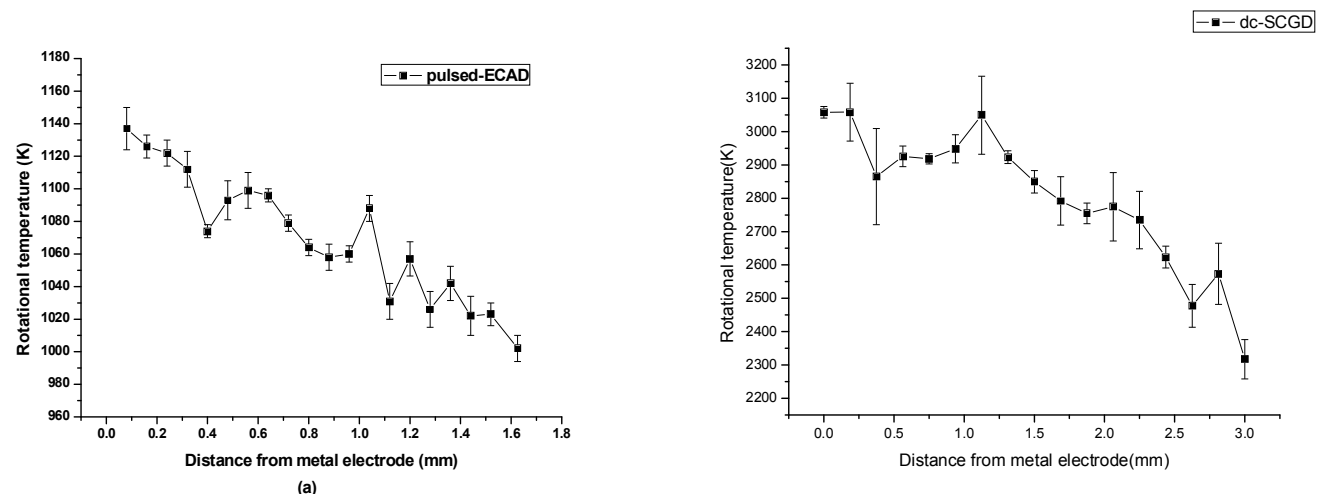

Fig. 10 The spatial distributions of the rotational temperatures of the pulsed-ECAD system and the dc-SCGD system

\subsection{Electron number density}

The electron number density plays an important role in understanding the plasma generation processes (excitation, ionization, etc.) [8]. Stark broadening $\left(\lambda_{\mathrm{S}}\right.$, in $\mathrm{nm}$ ) of the $\mathrm{H}_{\beta} 486.06 \mathrm{~nm}$ line was chosen for the determination of electron number densities $\left(n_{\mathrm{e}}\right.$, in $\left.\mathrm{cm}^{-3}\right)$ because it provides reasonable accuracy and precision with a relatively simple experimental setup and does not perturb the plasma or require an assumption of thermodynamic equilibrium[23]. To take the values of $\lambda_{s}$, experimental profiles of the $\mathrm{H}_{\beta}$ line, averaged for five consecutive measurements, were fitted to a Lorenz function using an Origin program (shown in Fig. 11). The Lorentzian part of full widths at half maximum (FWHMs) of the resulting line profiles was corrected for the instrumental broadening and used in the formula $\left(\lambda_{\mathrm{S}} / 4.800\right)=\left(n_{\mathrm{e}} / 10^{17}\right)^{0.68116}[34]$.

The spatial distributions of electron number densities $\left(n_{\mathrm{e}}\right.$, in $\left.\mathrm{cm}^{-3}\right)$ for the pulsed-ECAD and dc-SCGD systems were shown in Fig. 12. The electron number densities $n_{\mathrm{e}}$ of the pulsed-ECAD system, were measured within the range $(3.8-18.9) \times 10^{14} \mathrm{~cm}^{-3}$. Aleksandrov et al. [34] also measured the a the electron density in the high-voltage nanosecond discharge, and shown the electron density was 
varied between $10^{14}$ and $10^{15} \mathrm{~cm}^{-3}$, which was similar with our results. Compared to the results of Piotr Jamróz[8], which showed them in the range (3.4-6.8) $\times 10^{14} \mathrm{~cm}^{-3}$, the pulsed-ECAD system had higher electron number densities which indicated that the collision degree in the plasmas of pulsed-ECAD was more intense. The $n_{\mathrm{e}}$ of pulsed-ECAD at the near cathode region was higher than $n_{\mathrm{e}}$ at the near anode region by 5-6 times. However, the $n_{\mathrm{e}}$ could not be measured when the distance between metal and solution electrodes was more than $1 \mathrm{~mm}$. The same phenomenon was found in the dc-SCGD system. The electron number densities $n_{\mathrm{e}}$ of dc-SCGD were determined to be in the range $2.6 \times 10^{14} \sim 17.2 \times 10^{14} \mathrm{~cm}^{-3}$. The electron number densities of the two discharge systems stepped up with increasing distance from the metal electrode. Additionally, the behavior of electron number densities near $8.5 \times 10^{14} \mathrm{~cm}^{-3}$ for the negative glow and $2.5 \times 10^{14} \mathrm{~cm}^{-3}$ for the positive column found by Michael $\mathrm{R}$. Webb[23], was different from the space distribution of electron number densities of pulsed-ECAD, and its electron number densities relatively low.

The slightly higher electron number density in pulsed-ECAD than dc-SCGD plasma leads to the increase of electron collision frequency in pulsed-ECAD and the diminution of the electronic free path, which results in the diminution of electron energy obtained from the plasmas. Thus the excitation ability for heavy metal elements is weaker than dc-SCGD, and the reason maybe that only the spectra of the alkali metal and alkaline earth metal elements were occurred in the pulsed-ECAD system , because they need lower excitation power.

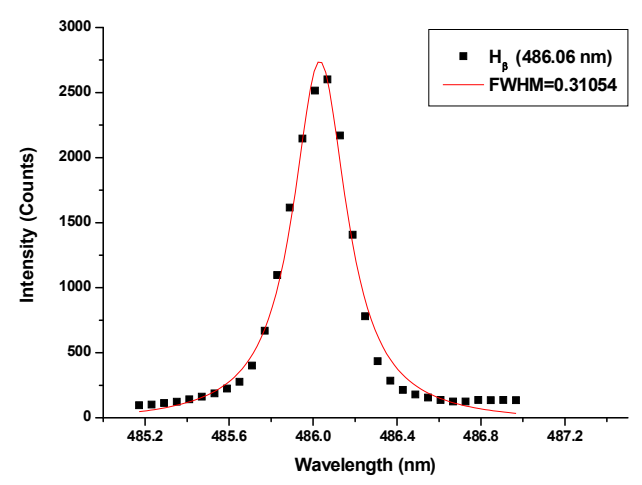

Fig. 11 Lorenz fitting graph of $\mathrm{H}_{\beta}(486.06 \mathrm{~nm})$
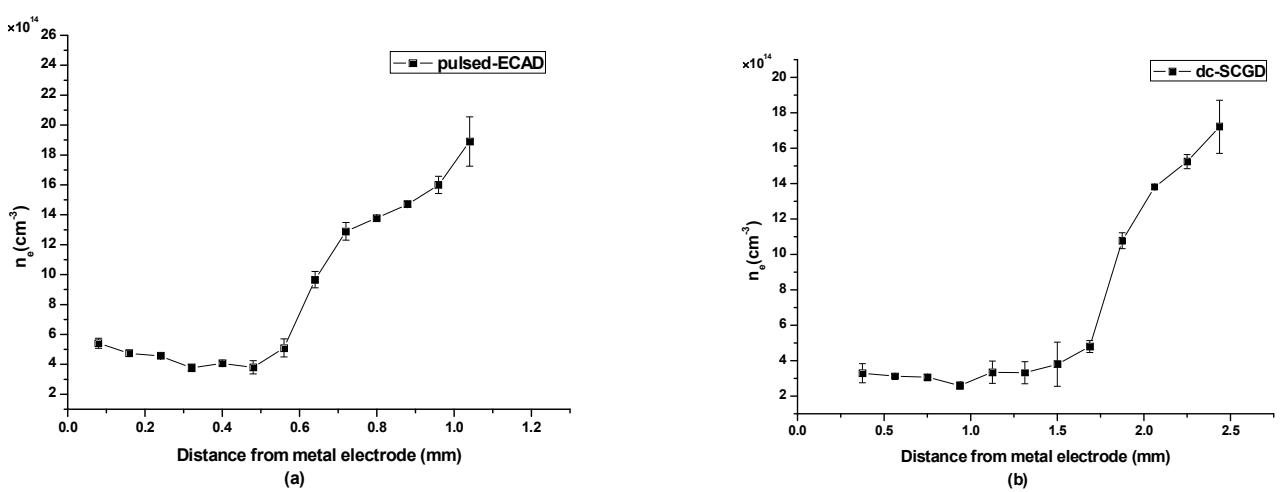

Fig. 12 Spatial distribution of electron number densities $\left(n_{\mathrm{e}}\right.$, in $\left.\mathrm{cm}^{-3}\right)$ of the pulsed-ECAD and dc-SCGD systems

\subsection{Influence of experimental parameters on plasma temperatures}

To better study the characteristics of the pulsed-ECAD plasmas, the dependences of the plasma 
temperatures on the experimental parameters, including discharge voltage and discharge frequency, were investigated.

\subsubsection{Effect of the discharge voltage}

In this part, the influence of the discharge voltage (peak voltage) on the excitation temperature of $\mathrm{H}$ atoms, the vibrational temperature of $\mathrm{N}_{2}$ and the rotational temperature of $\mathrm{OH}$ were studied (shown in Fig. 13). With the increasing discharge voltage, the pressure on the particles in the discharge plasma becomes larger, and the greater the acceleration the particles obtain. The particles get faster, which makes the collisions more intense and results in an increased emission intensity of the spectra. Thus, the plasma temperatures become higher. When the discharge voltage was more than $980 \mathrm{~V}$, the tungsten rod turns red and stray light was produced, which impacted the detection accuracy. A higher voltage also damaged the glass capillary tube and caused the discharge to become unstable [32].

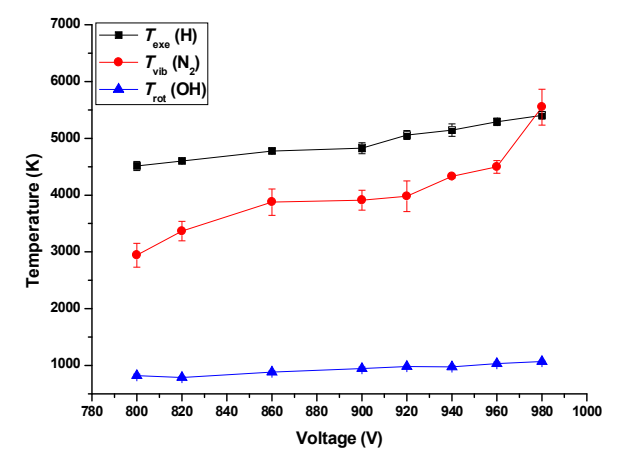

Fig. 13 Influence of discharge voltage on the plasma temperatures

\subsubsection{Influence of the discharge frequency}

The effect of discharge frequency on the excitation temperature of $\mathrm{H}$ atoms, the vibrational temperature of $\mathrm{N}_{2}$, the rotational temperature of $\mathrm{N}_{2}$ and $\mathrm{OH}$ was also investigated by collecting the emission spectra of the pulsed-ECAD system with various discharge frequencies. The results were presented in Fig. 14. When the frequency was more than $5.7 \mathrm{kHz}$, stable plasma cannot be obtained. With increasing discharge frequency, the acceleration time of electrons and ions may be shorter. The cycles of discharge voltage and current were reduced and the voltage reaches its maximum quickly. Thus, the time worked on the electrons and ions was shortened in one cycle, which caused weaker collisions and results in a decrease of plasma temperature. The reason for the absence of investigation of discharge frequencies lower than $4.7 \mathrm{kHz}$ is the limit of the power supply. Thus, it is possible that higher plasma temperatures could be obtained with a lower discharge frequency. 


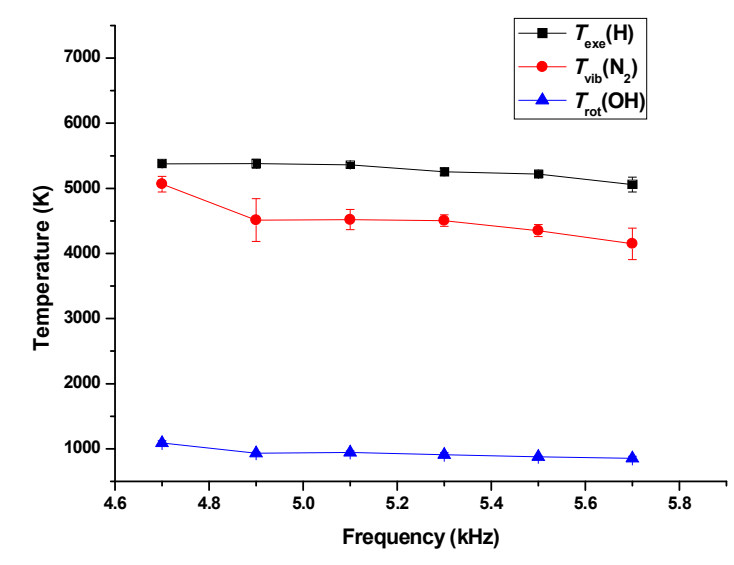

Fig. 14 Effect of discharge voltage on plasma temperatures

\section{Conclusions}

The temperature characteristics of the discharge plasmas of the source, sustained by an alternating current power supply coupled with a high voltage diode, were studied. The excitation temperature of $\mathrm{H}$ $T_{\text {exc }}(\mathrm{H})$, the vibrational temperature of $\mathrm{N}_{2} T_{\mathrm{vib}}\left(\mathrm{N}_{2}\right)$ and the rotational temperature of $\mathrm{OH} T_{\text {rot }}(\mathrm{OH})$ were measured to be $4900 \pm 36 \sim 6800 \pm 108 \mathrm{~K}, 4600 \pm 86 \sim 5800 \pm 100 \mathrm{~K}$ and $1050 \pm 20 \sim 1140 \pm 10 \mathrm{~K}$, respectively. According to previous experimental results, plasma temperatures of the pulsed-ECAD system are lower than the dc-SCGD, which means the thermal desolvation effect is weak. The effects of experimental conditions, including discharge voltage and frequency, on plasma temperatures were also explored. Plasma temperatures increased with the discharge increasing, and decreased with discharge frequency increasing. Additionally, the electron number density, as derived from the Stark broadening of the $\mathrm{H}_{\beta}$ line, was in the range $(3.8-18.9) \times 10^{14} \mathrm{~cm}^{-3}$. This was higher than the electron number density of the dc-SCGD system $\left(2.6 \times 10^{14} \sim 17.2 \times 10^{14} \mathrm{~cm}^{-3}\right)$, but the temperature lower than the dc-SCGD system, and it means that the high-energy particles of pulsed-ECAD system is less than the dc-SCGD system.

\section{Acknowledgements}

This work was financially supported by Key Project of Foundation and Advanced Technology Research Project of Chongqing (cstc2015jcyjBX0016), Project of Entrepreneurship and Innovation for Returned Overseas Scholars of Chongqing(cx2017126) and Foundation and Advanced Technology Research Project of Chongqing (cstc2016jcyjA0389).

\section{References}

1 Schwartz, A.J.;Ray, S.J.; Elish, E.; Storey, A.P.; Rubinshtein, A.A.; Chan, G.C. Visual observations of an atmospheric-pressure solution-cathode glow discharge.[J]. Talanta. 2012, 102:26-33.

2 Wang, J.; Tang, P, Zheng P, Zhai, X.; Analysis of metal elements by solution cathode glow discharge-atomic emission spectrometry with a modified pulsation damper[J]. J. Anal. At. Spectrom. 2017, 32:1925-1931.

3 Cserfalvi, T.; Mezei, P. Direct Solution Analysis by Glow Discharge: Electrolyte-Cathode Discharge Spectrometry[J]. J. Anal. At. Spectrom., 1994, 9:345-349.

4 Marcus, R.K.; Davis, W.C. An atmospheric pressure glow discharge optical emission source for the direct sampling of liquid media[J]. Anal. Chem. 2001, 73:2903-10. 
5 Manard, B.T; Koneggerkappel, S.; Gonzalez, J.J; Jose, C.; Meirong D.; Xiang, M.; Kenneth R.M.; Richard E.R. Liquid sampling-atmospheric pressure glow discharge as a secondary excitation source for laser ablation-generated aerosols: parametric dependence and robustness to particle loading.[J]. Appl. Spectrosc. 2015, 69:58-66.

6 Van K.D.; Kitano, A.; Yamamoto, T.; Ukita, Y.;Takamura, Y. Development of high sensitive liquid electrode plasma - Atomic emission spectrometry (LEP-AES) integrated with solid phase pre-concentration[J]. Microelectron. Eng. 2013, 111:343-347.

7 He, Q.; Zhu, Z.; Hu, S.; Zheng, H .; Jin, L. Elemental determination of microsamples by liquid film dielectric barrier discharge atomic emission spectrometry[J]. Anal. Chem, 2012, 84:4179-84.

8 Jamróz, P.; Żyrnicki, W. Spectroscopic Characterization of Miniaturized Atmospheric-Pressure dc Glow Discharge Generated in Contact with Flowing Small Size Liquid Cathode[J]. Plasma Chem Plasma Process. 2011,31:681-696.

9 Jamroz P, Pohl P and Zyrnicki W. An analytical performance of atmospheric pressure glow discharge generated in contact with flowing small size liquid cathode[J]. J Anal. At. Spectrom. 2012,27: 1032-1037.

10 Greda, K.; Jamróz, P.; Pohl, P. Effect of the addition of non-ionic surfactants on the emission characteristic of direct current atmospheric pressure glow discharge generated in contact with a flowing liquid cathode[J]. J Anal. At. Spectrom. 2013,28: 134-141

11 Greda, K.; P. Jamróz.; Pohl, P. The improvement of the analytical performance of direct current atmospheric pressure glow discharge generated in contact with the small-sized liquid cathode after the addition of non-ionic surfactants to electrolyte solutions[J]. Talanta. 2013,108: 74-82.

12 Webb MR, Andrade FJ and Gamez G. Spectroscopic and electrical studies of a solution-cathode glow discharge[J]. J Anal At Spectrom. 2005, 20:1218-1225

13 Aramendia, M.; Resano, M.; Vanhaecke, F. Determination of toxic trace impurities in titanium dioxide by solid sampling-electrothermal vaporization-inductively coupled plasma mass spectrometry[J]. $J$. Anal. At. Spectrom. 2008, 24:41-50.

14 Wang, Z.; Ni, ZM.; Qiu, DR.; Chen, T.Y.; Tao, G.Y.; Yang, P.Y. Determination of metal impurities in titanium dioxide using slurry sample introduction by axial viewing inductively coupled plasma optical emission spectrometry[J]. J. Anal. At. Spectrom. 2004,19:273-276.

15 Mottaleb, MA.; Yang, J.S.; Kim, H. Electrolyte-as-cathode glow discharge (elcad)/glow discharge electrolysis at the gas-solution interface[J]. Appl. Spectrosc. Rev.2002, 37:247-273

16 Webb, MR.; Hieje, GM. Spectrochemical Analysis by Using Discharge Devices with Solution Electrodes[J]. Anal. Chem. 2009, 81: 862-867

17 Greda, K.;Jamróz, P.; Dzimitrowicz, A.; Pohl, P. Direct elemental analysis of honeys by atmospheric pressure glow discharge generated in contact with a flowing liquid cathode[J]. J. Anal. At.Spectrom. 2014, 30:154-161

18 He, Q.; Zhu, Z.L.; Hu SH. Flowing and Nonflowing Liquid Electrode Discharge Microplasma for Metal Ion Detection by Optical Emission Spectrometry[J]. Appl. Spectrosc. Rev. 2014,49:249-269

19 Mezei, P.; Cserfalvi, T. Electrolyte Cathode Atmospheric Glow Discharges for Direct Solution Analysis[J]. Appl. Spectrosc. Rev. 2007,42:573-604.

20 Mezei, P.; Cserfalvi, T. A Critical Review of Published Data on the Gas Temperature and the Electron Density in the Electrolyte Cathode Atmospheric Glow Discharges[J]. Sensors, 2012, 12:6576.

21 Decker, C.G.; Webb, M.R. Measurement of sample and plasma properties in solution-cathode glow discharge and effects of organic additives on these properties[J]. J Anal At Spectrom. 2015,31:311-318

22 Cserfalvi, T.; Mezei, P.; Csillag, L. The spatial distribution of the temperatures and the emitted spectrum in the electrolyte cathode atmospheric glow discharge[J]. $J$ Phys D Appl Phys. 2005,38:2804-2811

23 Webb, M.R.; Andrade, F.J.; Hieftje, G.M. Use of electrolyte cathode glow discharge (ELCAD) for the analysis of complex mixtures[J]. J Anal At Spectrom 2007,22:766-774

24 Webb, MR.; George, C.; Chan, Y.; Andrade, F.J.; G. Gamez. Spectroscopic characterization of ion and electron populations in a solution-cathode glow discharge[J]. J. Anal. At. Spectrom. 2006, 21: 525-530

25 Bruggeman, P.; Liu, J.; Degroote, J. Dc excited glow discharges in atmospheric pressure air in pin-to-water electrode systems[J]. J Phys D Appl Phys. 2008, 41:2801-2809

26 Bruggeman, P.; Ribežl, E.; Maslani, A. Characteristics of atmospheric pressure air discharges with a liquid cathode and a metal anode[J]. Plasma Source Sci Technol. 2008, 17:431-438

27 Jamróz, P.; Żyrnicki, W.; Pohl, P. The effect of a miniature argon flow rate on the spectral characteristics of a direct current atmospheric pressure glow micro-discharge between an argon microjet and a small sized flowing liquid cathode[J]. Spectrochim Acta Part B. 2012,73:26-34 
28 Jamróz, P.; Gręda, K.; Pohl P. Direct Current Atmospheric Pressure Microdischarge Generated between a Miniature Flow Helium Microjet and a Flowing Liquid Cathode[J]. Plasma Process Polym. 2015, 11:755-762

29 Keiichiro, U.; Naoki, S.; Kentaro, T.; Tsuyoshi, A.; Tomoyuki, M. Diagnostics of atmospheric-pressure pulsed-dc discharge with metal and liquid anodes by multiple laser-aided methods[J]. Plasma Sources Sci. Technol. 2016, 25: 045004.

30 Li L, Nikiforov A and Xiong Q. Measurement of $\mathrm{OH}$ radicals at state X $2 \Pi$ in an atmospheric-pressure micro-flow dc plasma with liquid electrodes in $\mathrm{He}, \mathrm{Ar}$ and $\mathrm{N} 2$ by means of laser-induced fluorescence spectroscopy[J]. J Phys D Appl Phys. 2012, 45:18-26.

31 Zheng, P.C.; Chen, Y.Y.; and Wang, J.M. A pulsed atmospheric-pressure discharge generated in contact with flowing electrolyte solutions for metal element analysis by optical emission spectrometry[J]. J Anal At Spectrom. 2016, 31:2037-2044.

32 Zheng, P.C.; Wang, H.M.; Li, J.Q. Spectroscopic study of atmospheric pressure argon DC microdischarge[J]. Spectrosc Spect Anal. 2008, 28:2224-2227

33 Kohara, Y.; Terui, Y.; Ichikawa, M. Characteristics of liquid electrode plasma for atomic emission spectrometry[J]. J Anal At Spectrom. 2012,27:1457-1464

34 Aleksandrov NL, Kindysheva SV, Nudnova MMand Starikovskiy AY. Mechanism of ultra-fast heating in a non-equilibrium weakly ionized air discharge plasma in high electric fields[J]. J. Phys. D: Appl. Phys. 2010,43:255201. 